\title{
Evaluation of the Medical Care of Patients with Hypertension in an Emergency Department and in Ambulatory Hypertension Unit
}

\author{
Fernando Nobre, Fause Chauchar, Jaciara Machado Viana, Gustavo José Veras Pereira, \\ Nereida Kilza da Costa Lima \\ Ribeirão Preto, SP
}

\begin{abstract}
Objective - To evaluate the characteristics of the patients receiving medical care in the Ambulatory of $\mathrm{Hy}$ pertension of the Emergency Department, Division of Cardiology, and in the Emergency Unit of the Clinical Hospital of the Ribeirão Preto Medical School.
\end{abstract}

Methods - Using a protocol, we compared the care of the same hypertensive patients in on different occasions in the 2 different places. The characteristics of 62 patients, 29 men with a mean age of 57 years, were analyzed between January 1996 and December 1997.

Results - The care of these patients resulted in different medical treatment regardless of their clinical features and blood pressure levels. Thus, in the Emergency Unit, 97\% presented with symptoms, and $64.5 \%$ received medication to rapidly reduce blood pressure. In $50 \%$ of the cases, nifedipine $S L$ was the elected medication. Patients who applied to the Ambulatory of Hypertension presenting with similar features, or, in some cases, presenting with similar clinically higher levels of blood pressure, were not prescribed medication for a rapid reduction of blood pressure at any of the appointments.

Conclusion - The therapeutic approach to patients with high blood pressure levels, symptomatic or asymptomatic, was dependent on the place of treatment. In the Emergency Unit, the conduct was, in the majority of cases, to decrease blood pressure immediately, whereas in the Ambulatory of Hypertension, the same levels of blood pressure, in the same individuals, resulted in therapeutic adjustment with nonpharmacological management. These results show the need to reconsider the concept of hypertensive crises and their therapeutical implications.

Key words: hypertension, hypertensive crises, antihypertensive therapeutics

Hospital das Clínicas da Faculdade de Medicina de Ribeirão Preto - USP Mailing address: Fernando Nobre - Av. Bandeirantes, 3900 - 14049-900 - Ribeirão Preto, SP - Brazil - E-mail: fernando.nobre@uol.com.br
A hypertensive crisis is a clinical condition where a sudden increase in the level of blood pressure occurs, associated with signs, symptoms, or both, such as headache, recent visual alterations, vomiting, dizziness, and eye ground alterations, vasospasms, or target-organ damage, such as malignant hypertension, acute pulmonary edema, aortic dissection, stroke, or myocardial infarction ${ }^{1}$.

It is important to point out that it is not always easy to characterize symptoms as secondary to sudden elevation of blood pressure, because some situations, such as acute psychological stress, can be misleading especially in patients with many complaints. In these cases, suitable clinical evaluation is necessary, including a well-conducted anamnesis and a detailed physical examination.

Hypertensive crises are classified as hypertensive urgencies and emergencies ${ }^{2}$. In urgencies, the increase in blood pressure is associated with acute symptoms and does not present immediate risk to life or acute targetorgan damage; therefore, in these situations control of blood pressure must be done slowly within $24 \mathrm{~h}^{1}$. In hypertensive emergencies, the increase in blood pressure is followed by signs that indicate acute or ongoing target-organ damage or is life threatening. In these cases, patients should be admitted to the hospital and should receive fast-acting intravenous antihypertensive medication in an intensive care unit. Patients with very high blood pressure who are asymptomatic or have no targetorgan involvement must be treated ambulatorily because theirs is not a situation of hypertensive urgency or emergency ${ }^{3}$.

This study evaluates the peculiarities of the medical care given to the same hypertensive patients in 2 different situations: in the emergency unit when they are generally diagnosed as in hypertensive crisis and in the ambulatory hypertension unit at a routine visit, where they were identified as noncontrolled hypertensive patients. 


\section{Methods}

Sixty-two patients were retrospectively studied through a designed protocol, $29(47 \%)$ were male, with mean age of 57 years (ranging from 24 to 83 years), 39 patients $(63 \%)$ were married, $30(48 \%)$ were black. Twenty-five of them $(40 \%)$ were smokers and $42(70 \%)$ used antihypertensive medication, 12 (19\%) of them used it irregularly.

Of the 42 patients using medication, $52 \%$ used 1 medication, $40 \%$ were taking 2 drugs, and only $8 \%$ used 3 drugs.

Medical care in the Emergency Unit and Ambulatory of Hypertension was performed by physician interns, who were being trained at the Medical Clinic Division. The interns who were taking care of ambulatory patients are exclusively trained at the Division of Cardiology under strict supervision of the cardiologists.

Patients with a diagnosis of hypertensive emergency were excluded. The comparisons were made in those hypertensive patients that went to the Emergency Unit and were also attended at the Ambulatory of Hypertension after a few days.

We evaluated the medical care in the 2 different places and compared clinical patterns of presentation and the treatment proposed.

\section{Results}

Both the Emergency Unit and in the Ambulatory of Hypertension treated patients with several levels of blood pressure and patients with very high levels of systolic and diastolic blood pressure (tab. I).

Medications used in the emergency unit included isolated sublingual nifedipine $(50 \%)$, isolated sublingual captopril (12.5\%), oral propranolol (10\%), furosemide $(7.5 \%)$, nifedipine and captopril $(10 \%)$, nifedipine and propranolol $(7.5 \%)$, and clonidine $(5 \%)$.

\begin{tabular}{|c|c|c|c|c|c|c|}
\hline \multicolumn{7}{|c|}{$\begin{array}{l}\text { Table I - Distribution of the prevalence of blood pressure totals } \\
\text { obtained in patients receiving treatment in the emergency unit and the } \\
\text { ambulatory hypertension unit, according to the classifications of the } \\
\text { VI Joint National Committee on the Prevention, Detection, Evaluation, } \\
\text { and Treatment of High Blood Pressure }\end{array}$} \\
\hline \multirow[b]{2}{*}{ Classification } & \multirow[b]{2}{*}{ EU } & \multicolumn{2}{|c|}{ Treatment } & \multicolumn{3}{|c|}{ Treatment } \\
\hline & & Yes & No & $\mathrm{AH}$ & Yes & No \\
\hline \multicolumn{7}{|l|}{ SBP } \\
\hline$<140 \mathrm{mmHg}$ & $2(3 \%)$ & - & $2(100 \%)$ & $15(24 \%)$ & $*$ & $*$ \\
\hline$\geq 140-159 \mathrm{mmHg}$ & $17(28 \%)$ & $7(42 \%)$ & $10(58 \%)$ & $20(32 \%)$ & $*$ & * \\
\hline$\geq 160-178 \mathrm{mmHg}$ & $20(32 \%)$ & $13(65 \%)$ & $7(35 \%)$ & $15(24 \%)$ & $*$ & * \\
\hline$\geq 180 \mathrm{mmHg}$ & $23(37 \%)$ & $16(69 \%)$ & $7(31 \%)$ & $12(20 \%)$ & * & * \\
\hline \multicolumn{7}{|l|}{ DBP } \\
\hline$<90 \mathrm{mmHg}$ & $3(5 \%)$ & $1(33,3 \%)$ & $2(66,6 \%)$ & $12(19 \%)$ & * & * \\
\hline$\geq 90-99 \mathrm{mmHg}$ & $20(32 \%)$ & $8(40 \%)$ & $12(60 \%)$ & $19(31 \%)$ & $*$ & $*$ \\
\hline$\geq 100-109 \mathrm{mmHg}$ & $20(32 \%)$ & $12(60 \%)$ & $8(40 \%)$ & $20(32 \%)$ & $*$ & $*$ \\
\hline$\geq 110 \mathrm{mmHg}$ & $19(31 \%)$ & $19(100 \%)$ & - & $11(17 \%)$ & * & * \\
\hline Total & $62(100 \%)$ & & & $62(100 \%)$ & $*$ & $*$ \\
\hline
\end{tabular}

Ambulatory patients were not medicated to rapidly reduce blood pressure, even though some of them had high blood pressure levels. On the other hand, patients in the Emergency Unit received treatment to rapidly reduce blood pressure, even those with discreet elevations, which reveals the inadequacy of criteria in the use of antihypertensive medication.

In the Emergency Unit, all patients who presented with diastolic blood pressure $\geq 110 \mathrm{mmHg}$ ( 19 pacientes) always received medication, regardless of their clinical condition.

Of the 62 patients sent to the Emergency Unit, 60 (97\%) were symptomatic, and of them, $40(64.5 \%)$ received treatment for rapid reduction of blood pressure (tab. II). The most frequent signs of presentation were: headache (84\%), fainting (77\%), psychomotor agitation (74\%), and others such as chest pain, nausea, lippitude and malaise in smaller percentages. In many cases, the presenting symptom, such as headache, may have been the cause of the blood pressure elevation and not its consequence.

Only 2 asymptomatic patients did not receive medication in the Emergency Unit. Sixteen of 62 patients who presented to the Ambulatory of Hypertension were asymptomatic and did not receive any medication, as mentioned above.

\section{Discussion}

The present study indicates how often patients arrive at the Emergency Unity and are inappropriately diagnosed as having a hypertensive crisis. They are also medicated as such, representing $64.5 \%$ of the patients studied.

On the other hand, no patient received antihypertensive medication in the Ambulatory of Hypertension, even though $26 \%$ of them were symptomatic and approximately $40 \%$ were in stage III of VI JNC. In the Emergency Unit, patients are probably receiving unnecessary and abusive medications, which may lead to undesirable side effects, increasing morbidity and mortality.

It is necessary to emphasize the concept of the hypertensive crisis and its therapeutical implications. Distinguishing patients with hypertensive crises is important to the viability of hypertensive emergency units, which usually receive a large number of patients unnecessarily medicated.

Although this study was performed in a hospital linked with a medical school of a major university with high standards of teaching and concept, a clear discrepancy exists between the correct concept and the treatment administrated. The most frequently used medication was

\begin{tabular}{|c|c|c|c|c|}
\hline \multicolumn{5}{|c|}{$\begin{array}{c}\text { Table II - Treatment for immediate reduction of blood pressure with } \\
\text { antihypertensive medication and symptoms }\end{array}$} \\
\hline \multirow[b]{2}{*}{ Treatment } & \multicolumn{2}{|c|}{ Emergency Unit } & \multicolumn{2}{|c|}{ Ambulatory of Hypertension } \\
\hline & Symptomatic & Asymptomatic & Symptomatic & Asymptomatic \\
\hline & $(60)$ & (2) & (16) & (46) \\
\hline Yes & $40(64.5 \%)$ & - & - & - \\
\hline No & $20(35.5 \%)$ & $2(3 \%)$ & $16(26 \%)$ & $46(74 \%)$ \\
\hline Total & $60(97 \%)$ & $2(3 \%)$ & $16(26 \%)$ & $46(74 \%)$ \\
\hline
\end{tabular}


nifedipine SL, but the indiscriminate use of this medication as well as other antihypertensive medications is not recommended ${ }^{3,4}$.

In elderly patients or in patients with coronaryopathies, the indiscriminate use of antihypertensive medication can be deleterious, leading to serious or even irreversible complications, due to the difficulty in gradually reducing blood pressure levels ${ }^{5-8}$.

Therefore, the treatment of patients with high levels of blood pressure, symptomatic or not, depends on the medical care unit used. When attended at the emergency unit, the recommendation was, in general, immediate reduction of blood pressure, but in the ambulatory hypertension unit, when similar levels of blood pressure were observed, the recommendation was therapeutical adjustment and the use of nonmedication measures. It is clearly necessary to reconsider the concept of hypertensive crises and their therapeutical implications.

\section{References}

1. Joint National Committee on Detection, Evaluation and Treatment of High Blood Pressure. The Sixth Report of the Joint National Committee on the Prevention, Detection, Evaluation, and Treatment of High Blood Pressure. Arch Intern Med 1997; 157: 2413-46.

2. Ram CVS. Hypertensive Crises. In: Kaplan NM, Ram CVS, ed. Individualized Therapy of Hypertension, $1^{\text {st }}$ ed. New York: Marcel Dekker Inc, 1995: 223-44.

3. Kaplan NM. Clinical Hypertension, $7^{\text {th }}$ ed. Baltimore: Williams and Wilkins, 1998: 265-80.

4. Leeman M. Use of antyhipertensive drugs in an intensive care unit. Am J Hypertens 1997; 10: 144.
5. Zampaglione B, Pascale C, Marchisio M, Cavallo-Perin P. Prevalence and clinical presentation. Hypertension 1996; 27: 144-7.

6. Yussuf S. Calcium antagonists in coronary artery disease and hypertension: time for reevaluation? Circulation 1995; 92: 1079-82.

7. Stason WB, Schmid CH, Niedzwiecki D, et al. Safety of nifedipine in patients with hypertension: a meta-analysis. Hypertension 1997; 30(part 1): 7-14.

8. Opie LH, Messerli FH. Nifedipine and mortality: grave defects in the dossier. Circulation 1995; 92: 1068-73. 